\title{
Opsanus brasiliensis Rotundo, Spinelli \& Zavalla-Camin, 2005 (Teleostei: Batrachoidiformes: Batrachoididae), sinônimo-júnior de Opsanus beta (Goode \& Bean, 1880), com notas sobre a ocorrência da espécie na costa brasileira
}

\author{
Rodrigo Antunes Caires ${ }^{1}$, Helen Audrey Pichler ${ }^{2}$, Henry Louis Spach² \& Juliana Martinhão Ignácio ${ }^{3}$
}

Biota Neotropica v7 (n2) - http://www.biotaneotropica.org.br/v7n2/pt/abstract?article+bn02307022007

\author{
Recebido em 26/02/07 \\ Versão Reformulada recebida em 28/04/07 \\ Publicado em 08/06/07 \\ ${ }^{1}$ Museu de Zoologia, Universidade de São Paulo - USP, \\ CP 42694, CEP 04263-000, São Paulo, SP, Brasil \\ ${ }^{2}$ Programa de Pós-Graduação em Ciências Biológicas - Zoologia, \\ Universidade Federal do Paraná - UFPR, CP 19020, CEP 81531-980, Curitiba, Paraná, Brasil \\ ${ }^{3}$ Programa de Pós-Graduação em Sistemas Costeiros e Oceânicos, \\ Universidade Federal do Paraná - UFPR, CP 50002, CEP 83255-000, Pontal do Paraná, Paraná, Brasil \\ Autor para correspondência: Rodrigo Antunes Caires, e-mail: rodricaires@yahoo.com.br
}

\section{Abstract}

Caires, R.A., Pichler, H.A., Spach, H. L. \& Ignácio, J.M. Opsanus brasiliensis Rotundo, Spinelli \& ZavallaCamin, 2005 (Teleostei: Batrachoidiformes: Batrachoididae), a junior synonym of Opsanus beta (Goode \& Bean, 1880), with notes on its occurrence in the Brazilian coast. Biota Neotrop. May/Aug 2007 vol. 7, no. 2. http://www.biotaneotropica.org.br/v7n2/pt/abstract?article+bn02307022007. ISSN 1676-0603.

In this paper, two individuals of Opsanus caught in the coast of Paraná (the first records of this genus in the southern Brazil) were identified as Opsanus beta (Goode \& Bean, 1880). Such speciemens were compared with the type series of Opsanus brasiliensis Rotundo, Spinelli \& Zavalla-Camin, 2005 (originally described from the coast of Santos, São Paulo), and with the literature data. As a result, Opsanus brasiliensis is herein regarded as a junior synonym of $O$. beta, and so the exemplars of Opsanus found at the Brazilian coast are exotic. It is herein postulated that $O$. beta would have invaded the Brazilian coast through ballast water released by vessels that sailed from the natural areas of occurrence of such species.

Keywords: Opsanus beta, taxonomy, distribution.

\section{Resumo}

Caires, R.A., Pichler, H.A., Spach, H. L. \& Ignácio, J.M. Opsanus brasiliensis Rotundo, Spinelli \& ZavallaCamin, 2005 (Teleostei: Batrachoidiformes: Batrachoididae), sinônimo-júnior de Opsanus beta (Goode \& Bean, 1880), com notas sobre a ocorrência da espécie na costa brasileira. Biota Neotrop. May/Aug 2007 vol. 7, no. 2. http://www.biotaneotropica.org.br/v7n2/pt/abstract?article+bn02307022007. ISSN 1676-0603.

Neste trabalho, dois exemplares de Opsanus coletados na costa do Paraná, e que representam os primeiros registros do gênero no sul do Brasil, foram identifcados como Opsanus beta (Goode \& Bean, 1880). Uma comparação mais detalhada entre estes exemplares e a série-tipo de Opsanus brasiliensis Rotundo, Spinelli \& Zavalla-Camin, 2005 (descrita originalmente do litoral de Santos, São Paulo), além de informações da literatura, indicou que Opsanus brasiliensis é sinônimo-júnior de Opsanus beta e que os representantes de Opsanus aqui encontrados são exóticos. É aqui levantada a possibilidade de que $O$. beta invadiu a costa brasileira através de água de lastro de navios que teriam zarpado das áreas naturais de ocorrência desta espécie.

Palavras-chave: Opsanus beta, taxonomia, distribuição. 


\section{Introdução}

De acordo com informações da literatura mais recente (Menezes \& Figueiredo 1998, Carvalho-Filho, 1999, Menezes et al. 2003), a Família Batrachoididae é representada por seis gêneros e treze espécies na costa brasileira, quatro das quais (Porichthys porosissimus, Thalassophryne montevidensis, T. nattereri e Triathalassotia lambaloti) no sudeste-sul do Brasil (do Espírito Santo à Argentina).

Há dois anos, no entanto, foram coletados nas cercanias de Santos, SP, vários exemplares de outro gênero, Opsanus, distinto dos demais representantes de Batrachoididae aqui encontrados pela presença de um poro na axila da nadadeira peitoral, além de várias glândulas de muco nesta região do corpo. Até então, as quatro espécies conhecidas deste gênero haviam sido registradas apenas no Atlântico Norte-Ocidental (Robins et al. 1986, McEachran \& Fechhelm 1998, Collette 2002). Este material foi examinado por Rotundo et al. (2005) que, com base em diferenças de coloração e de contagem de vértebras, descreveram uma espécie nova, Opsanus brasiliensis.

Mais recentemente, entre março e julho de 2006, dois espécimes deste gênero foram coletados na costa do Paraná, um dos quais na Baía de Antonina, e o outro no litoral do Maciel, Pontal do Paraná (Figura 1). A comparação deste material com as informações da literatura (Collette, 2001, 2002) não deixou dúvidas de que estes exemplares pertencem à espécie Opsanus beta (Goode \& Bean,
1880). Neste trabalho, os dados destes espécimes são comparados com material de Opsanus beta proveniente da Flórida, e com todo o material-tipo de Opsanus brasiliensis, que está depositado no Museu de Zoologia da Universidade de São Paulo (MZUSP).

\section{Material e Métodos}

Os exemplares coletados no litoral do Paraná foram catalogados e depositados no Museu de Zoologia da USP. Todos os exemplares foram examinados segundo Collette $(1974,2001)$. As medidas foram obtidas através de paquímetro digital, arredondadas para o décimo de milímetro mais próximo $(0,1 \mathrm{~mm})$, e analisadas em função do comprimento padrão $(\mathrm{CP})$. Para a comparação preliminar da contagem de vértebras, foram feitas radiografias (Radiógrafo Faxitron MX-20) de um dos exemplares coletados no Paraná (MZUSP 92420), e do material proveniente da Flórida (MZUSP 47294). O esqueleto de um indivíduo de Opsanus coletado em Santos foi preparado, e a contagem de vértebras foi comparada com os valores obtidos através das radiografias. O material examinado encontra-se listado abaixo. O número de exemplares por lote e a amplitude de variação do comprimento padrão estão indicados entre parênteses. $\mathrm{O}$ espécime cujo esqueleto foi preparado está marcado com um asterisco (*).

Material examinado: ESTADOS UNIDOS, FLÓRIDA: MZUSP 47294, Cedar Keys, around Atsena Otey Bay, 12.ix.1950 (G.K. Reid) (4ex.; 30,3-126,6 mm). BRASIL, SÃO PAULO: MZUSP 88072,

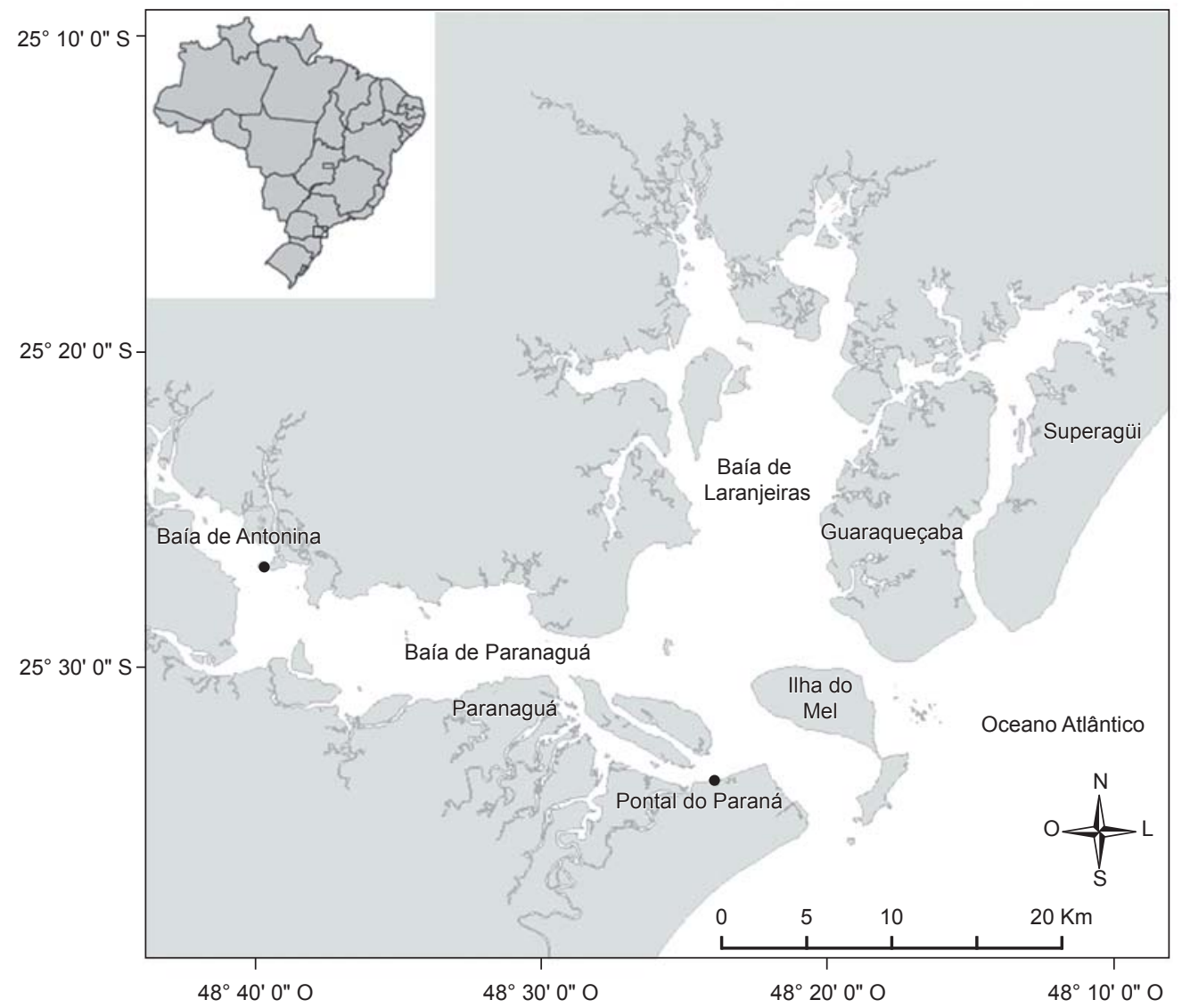

Figura 1. Mapa do Complexo Estuarino de Paranaguá (Paraná, Brasil), onde se localizam a Baía de Antonina e o infralitoral raso do Maciel (Pontal do Paraná), indicando os locais onde foram coletados os dois exemplares de Opsanus beta em 2006 (círculos pretos).

Figure 1. Map of the estuarine complex of Paranaguá (State of Paraná, Brazil), where the Antonina Bay and the shallow infrallitoral zone of Maciel (Pontal do Paraná) is located, indicating the sites where two Opsanus beta specimens were caught in 2006 (black circles). 
Santos, 23 59' 06" S e 46 $16^{\circ}$ '42" S, 07.vii.2004 (N. Camargo) (10 ex.; 113,4-205,9 mm). MZUSP 88073, Santos, 23 59' 06” S e 46 18' 42" S, 10.xi. 2004 (M. Croce) (4ex.; 109,2-223,0 mm). MZUSP 88074, Santos, 23 59'06" S e 46 18'42” S, 14.xi.2004 (M. Croce) (6ex.; 173,9-247,0 mm). MZUSP 88075, Santos, $23^{\circ} 58^{\prime} 12^{\prime}$ ' S e $46^{\circ} 20^{\prime}$ 54" W, 7.i.2005 (M. Croce) (3ex.; 189,7213,0 mm). MZUSP 88076, Santos, $2^{\circ}$ 59' 06” S $46^{\circ} 18^{\prime} 42^{\prime \prime} \mathrm{S}$, 03.ii.2005 (M. Croce) (4ex.; 118,7-193,1 mm). MZUSP 88077, Santos, $23^{\circ} 59^{\prime} 06^{\prime \prime} \mathrm{S} 46^{\circ} 18^{\prime} 42^{\prime \prime} \mathrm{S}, 15$. iii.2005 (M. Croce) (4ex.; 81,5-145,6 mm). MZUSP 88078, Santos, 23 59'06” S 46 18' 42”' W, 15.iii.2005 (M. Croce) (holótipo de Opsanus brasiliensis; 242,0 mm). MZUSP 89580, Porto de Santos, 25.iv.2005 (M. Itagaki) (2ex.; 172,7-200,0* mm). BRASIL, PARANÁ: MZUSP 92420, Antonina, 2526' 43" S e 48 39' 58' W, 08.iii.2006 (col.: H.A. Pichler) (1ex; $107,3 \mathrm{~mm}$ ). MZUSP 93288, infralitoral raso do Maciel, $25^{\circ} 33^{\prime} 14^{\prime \prime} \mathrm{S}$ e 48²4’06” W, 05.vii.2006 (col.: J.M. Ignácio) (1ex.; 250,0 mm).

\section{Resultados e Discussão}

Como pode ser observado nas Tabelas 1 a 3, há sobreposição de todos os caracteres merísticos e morfométricos entre os exemplares de Opsanus beta da Flórida, os exemplares de O. beta do Paraná, e a série-tipo de Opsanus brasiliensis. Estes valores estão dentro da variação apresentada na literatura sobre Opsanus beta (Collette, 2001, 2002). Rotundo et al. (2005) enfatizaram a diferença do número de vértebras pré-caudais entre os exemplares de Opsanus brasiliensis, que teriam nove elementos, e os demais representantes do gênero (com dez ou onze elementos), como um dos caracteres diagnósticos desta espécie. Entretanto, é provável que os autores tenham deixado de contar a primeira vértebra pré-caudal. Como já havia enfatizado Collette (2001), a crista supraocciptal é muito desenvolvida nas espécies deste gênero e cobre o primeiro supraneural, levando a uma subestimativa da contagem de vértebras em radiografias, erro no qual o próprio Collette havia incorrido anteriormente (2001, p. 2). De fato, foram observadas onze vértebras pré-caudais na preparação de esqueleto de um exemplar de Opsanus proveniente de Santos (Figura 2), um valor maior que o obtido nas radiografias (dez), e equivalente ao valor encontrado nos indivíduos de Opsanus beta (ver Collette, 2001).

O padrão de colorido do corpo é uma informação extremamente importante para a diagnose das espécies de Opsanus, sendo que $O$. beta apresenta um padrão de colorido de manchas ovais claras

Tabela 1. Contagens de raios das nadadeiras dorsal, anal e peitoral em exemplares de Opsanus beta (MZUSP 47294, exemplares coletados no litoral do Paraná, e informações da literatura), e nos exemplares da série-tipo de $O$. brasiliensis. Valores referem-se ao número de exemplares com a característica. Dados da literatura como em Collette (2001).

Table 1. Counts of dorsal, anal, and pectoral fin rays in individuals of Opsanus beta (MZUSP 47294, specimens caught in the Paraná coast, and literature data), and in the type series of $O$. brasiliensis. Values indicate the number of specimens with the aforementioned character. Literature data as Collette (2001).

\begin{tabular}{|c|c|c|c|c|c|c|c|c|c|c|}
\hline & \multicolumn{3}{|c|}{ Raios da dorsal } & \multicolumn{3}{|c|}{ Raios da anal } & \multicolumn{4}{|c|}{ Raios da peitoral } \\
\hline & 24 & 25 & 26 & 20 & 21 & 22 & 17 & 18 & 19 & 20 \\
\hline O. beta (Flórida) & - & 3 & 1 & - & - & 4 & - & 2 & 4 & - \\
\hline O. beta (Paraná) & - & 1 & - & - & 1 & - & - & - & 2 & - \\
\hline O. beta (literatura) & 1 & 7 & - & 1 & 6 & 1 & - & 5 & 3 & - \\
\hline O. brasiliensis & 2 & 4 & 2 & - & 6 & 3 & 1 & 15 & 1 & 4 \\
\hline
\end{tabular}

Tabela 2. Contagens das papilas da linha lateral e das glândulas da axila da peitoral em exemplares de Opsanus beta (MZUSP 47294, exemplares coletados no litoral do Paraná, e informações da literatura) e na série-tipo de $O$. brasiliensis. Notações dos valores como na Tabela 1.

Table 2. Counts of lateral line papillae and pectoral axil glands in specimens of Opsanus beta (MZUSP 47294, specimens caught in Paraná coast, and literature data) and in individuals from the type series of $O$. brasiliensis. Notations as in Table 1.

\begin{tabular}{|c|c|c|c|c|c|c|c|c|c|c|c|c|}
\hline \multicolumn{13}{|c|}{ Papilas da linha lateral dorsal } \\
\hline & 23 & 24 & 25 & 26 & 27 & 28 & 29 & 30 & & & & \\
\hline O. beta (Flórida) & - & - & 1 & 1 & 1 & 2 & - & - & & & & \\
\hline O. beta (Paraná) & 1 & 1 & 1 & - & - & - & - & - & & & & \\
\hline O. beta (literatura) & - & - & 3 & - & 2 & 1 & 1 & - & & & & \\
\hline O. brasiliensis & 2 & 6 & 8 & 13 & 13 & 11 & 5 & 2 & & & & \\
\hline \multicolumn{13}{|c|}{ Papilas da linha lateral ventral } \\
\hline & 18 & 19 & 20 & 21 & 22 & 23 & 24 & 25 & 26 & 27 & 28 & 29 \\
\hline O. beta (Flórida) & - & - & - & - & - & 1 & 1 & 2 & 1 & - & - & - \\
\hline O. beta (Paraná) & - & - & - & 1 & 2 & - & - & - & - & - & - & - \\
\hline O. beta (literatura) & - & - & - & - & 3 & 1 & 1 & 2 & - & - & - & - \\
\hline O. brasiliensis & 1 & 2 & - & 6 & 13 & 12 & 5 & 7 & 1 & - & 1 & - \\
\hline \multicolumn{13}{|c|}{ Glândulas da axila da peitoral } \\
\hline & 4 & 5 & 6 & 7 & 8 & 9 & 10 & 11 & 12 & 13 & 14 & 15 \\
\hline O. beta (Flórida) & - & - & - & - & 1 & 1 & 2 & 1 & 1 & - & - & - \\
\hline O. beta (Paraná) & - & - & - & - & - & - & 2 & 1 & - & - & - & - \\
\hline O. beta (literatura) & - & - & 1 & - & 2 & 1 & 1 & 3 & 1 & - & - & - \\
\hline O. brasiliensis & 5 & 5 & 3 & 13 & 12 & 10 & 4 & 3 & - & - & - & - \\
\hline
\end{tabular}


Tabela 3. Dados morfométricos dos exemplares de Opsanus beta do Atlântico Norte-Ocidental (Estados Unidos, Flórida), da série típica de Opsanus brasiliensis, e dos exemplares de Opsanus coletados no litoral do Paraná (Antonina e litoral de Maciel). Valores do comprimento padrão em milímetros, e proporções do corpo em partes por mil do comprimento padrão (\%oCP). Dados da literatura como em Collette (2001. Tabela 1).

Table 3. Morphometric data of the Opsanus beta specimens from the western North Atlantic (United States, Florida), the type-series of Opsanus brasiliensis, and the individuals of Opsanus collected in the coast of Paraná (Antonina and littoral of Maciel). Values of standard length in milimeters, and proportional data expressed in thousandths of the standard length $(\%$ CP). Literature data as Collette (2001. Table 1)

\begin{tabular}{|c|c|c|c|c|c|c|c|}
\hline & \multicolumn{2}{|c|}{$\begin{array}{c}\text { Opsanus beta } \\
\text { Flórida }(\mathbf{N}=4)\end{array}$} & \multicolumn{2}{|c|}{$\begin{array}{c}\text { Opsanus brasiliensis } \\
\text { parátipos }(\mathbf{N}=30)\end{array}$} & \multirow[t]{2}{*}{$\begin{array}{l}\text { O. brasiliensis } \\
\text { (holótipo) }\end{array}$} & \multirow[t]{2}{*}{$\begin{array}{c}\text { Opsanus beta } \\
\text { Paraná }\end{array}$} & \multirow[t]{2}{*}{$\begin{array}{c}\text { Opsanus beta } \\
\text { (literatura) }\end{array}$} \\
\hline & Mín. & Máx. & Mín. & Máx. & & & \\
\hline Comprimento padrão (mm) & 30,3 & 126,6 & 81,5 & 247,0 & 242,0 & $107,3-200,5$ & $77,5-200,0$ \\
\hline \multicolumn{8}{|l|}{$\% \circ \mathrm{CP}$} \\
\hline Comprimento da cabeça & 297,8 & 387,4 & 357,3 & 412,4 & 358,5 & $360,0-390,9$ & $367,0-412,0$ \\
\hline Largura máxima da cabeça & 229,2 & 297,4 & 273,3 & 346,0 & 326,0 & $268,8-292,4$ & $246,0-324,0$ \\
\hline Altura máxima da cabeça & 83,6 & 228,6 & 197,2 & 271,5 & 250,9 & $191,6-221,3$ & \\
\hline Comprimento orbital & 49,5 & 104,5 & 41,7 & 68,4 & 42,1 & $49,8-64,0$ & $63,0-92,0$ \\
\hline Largura interorbital & 37,6 & 52,3 & 41,3 & 69,4 & 62,1 & $54,5-57,1$ & $54,0-86,0$ \\
\hline Distância pré-dorsal & 437,0 & 584,8 & 447,2 & 568,4 & 512,1 & $351,3-478,1$ & $471,0-486,0$ \\
\hline Distância pré-anal & 533,1 & 585,5 & 560,6 & 635,5 & 625,7 & $576,6-612,7$ & $551,0-600,0$ \\
\hline Comprimento da peitoral & 203,1 & 253,2 & 173,3 & 253,7 & 194,1 & $222,9-224,4$ & $196,0-223,0$ \\
\hline Comprimento da pélvica & 149,0 & 213,3 & 124,4 & 185,2 & 147,3 & $157,4-173,3$ & $160,0-182,0$ \\
\hline
\end{tabular}

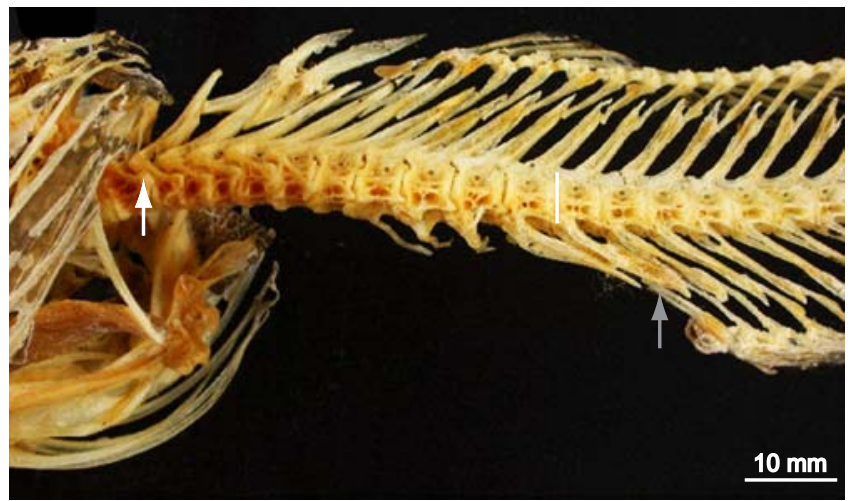

Figura 2. Vista lateral do esqueleto cranial (porção posterior) e do esqueleto axial de Opsanus beta (MZUSP 89580, 200,0 mm CP). Seta branca indica a primeira vértebra pré-caudal, e a barra vertical branca indica o espaço entre a última vértebra pré-caudal e a primeira vértebra caudal, que porta o pterigióforo do primeiro raio da nadadeira anal (seta cinza).

Figure 2. Lateral view of the cranial skeleton (posterior portion) and the axial skeleton of Opsanus beta (MZUSP 89580, $200.0 \mathrm{~mm} \mathrm{CP}$ ). White arrow indicates the first pre-caudal vertebra, and white vertical bar, the space between the last pre-caudal vertebra and the first caudal vertebra, which bears the first anal fin pterygiophore (gray arrow).

discretas na nadadeira peitoral, manchas claras em forma de roseta na região lateral do tronco, e a mucosa bucal de cor branco-leitosa, com uma faixa escura estreita atrás das séries de dentes do dentário (Schultz \& Reid, 1937, Collette, 2001, 2002). Todas estas características diagnósticas do colorido de Opsanus beta são encontradas nos espécimes coletados na costa do Paraná, e também no holótipo de Opsanus brasiliensis (Figura 3). Rotundo et al. (2005) confundem-se ao tentar diferenciar a coloração de $O$. brasiliensis da de $O$. beta, pois afirmam que esta última espécie difere da espécie por eles descrita na presença de "manchas claro-escuras irregulares" (p. 96), mas descrevem a coloração de Opsanus brasiliensis empregando esta mesma frase (p. 95).

Em suma, não existem motivos para considerar Opsanus brasiliensis Rotundo et al. como uma espécie diferente de Opsanus
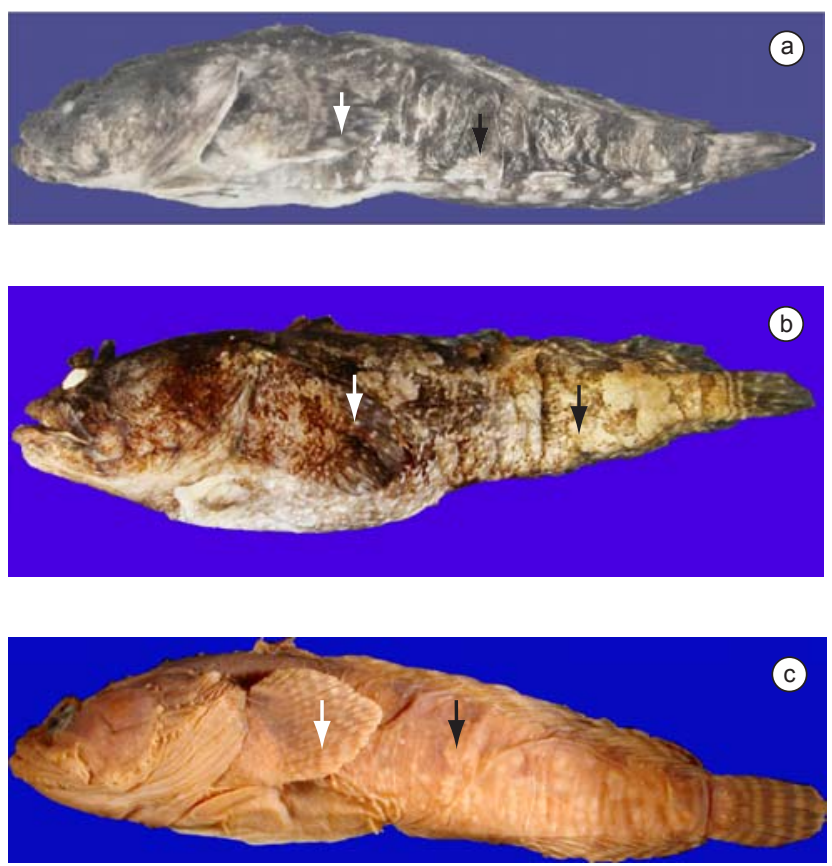

Figura 3. a) Opsanus beta, MZUSP $92420,107,3 \mathrm{~mm}$ de comprimento padrão. Paraná, Antonina, $25^{\circ} 26^{\prime} 43^{\prime \prime} \mathrm{S}$ e 48 $38^{\circ} 58^{\prime \prime}$ W; b) Opsanus beta, MZUSP 88078, holótipo de $O$. brasiliensis, $242,0 \mathrm{~mm}$ de comprimento padrão e c) Opsanus beta, MZUSP 47294, 126,6 mm de comprimento padrão, Estados Unidos, Flórida, Cedar Keys, nas proximidades de Atsena Otey Bay. Observe que todos estes exemplares apresentam as manchas ovais claras e discretas na nadadeira peitoral (setas brancas), e as grandes manchas claras em forma de roseta nos flancos (setas pretas).

Figure 3. a) Opsanus beta, MZUSP 92420, $107.3 \mathrm{~mm}$ of standard length,

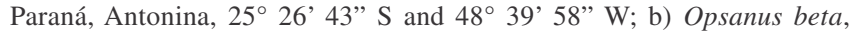
MZUSP 88078, holotype of $O$. brasiliensis, $242.0 \mathrm{~mm}$ of standard length. and c) Opsanus beta, MZUSP 47294, $126.6 \mathrm{~mm}$ of standard length, United States, Florida, Cedar Keys, around Atsena Otey Bay. Observe that all these specimens present pale round spots on the pectoral fin (white arrows), and large pale rosettes on the flanks (black arrows). 
beta (Goode \& Bean, 1880). A descrição original de Opsanus brasiliensis não indica a localidade-tipo, tampouco os números de registro dos parátipos. Estas informações constam da presente lista do material examinado.

Opsanus beta distribui-se originalmente no Golfo do México, de Belize até Palm Beach, na Flórida (Collette, 2002). Os recentes registros desta espécie na costa sudeste-sul do Brasil, em locais tão distantes da sua área de distribuição, indicam que ela é exótica no Atlântico Sul-Ocidental. No momento não é possível saber como ela teria chegado aqui, mas, uma vez que os registros desta espécie na costa brasileira estão próximos de zonas portuárias, é possível que Opsanus beta tenha invadido estas regiões através de água de lastro despejada por navios que zarparam das áreas naturais de ocorrência desta espécie (Golfo do México ou costa dos Estados Unidos). Há vários casos de invasão de organismos marinhos no Brasil através de água de lastro (Silva \& Souza, 2004), inclusive de peixes, como foi o caso de uma espécie de blênio nativa do Indo-Pacífico, Omobranchus punctatus (Valenciennes, 1836), que foi registrada nas costas da Bahia, Rio de Janeiro e Santa Catarina (Gerhardinger et al. 2006). Por sua vez, uma espécie de peixe marinho nativa do Brasil (Pinguipes brasilianus Cuvier, 1829) foi introduzida via água de lastro na costa da Itália (Orsi Relini, 2002).

Os dois exemplares de Opsanus beta coletados no Paraná representam os primeiros registros desta espécie no litoral sul do Brasil. No litoral de Santos, esta espécie parece ser freqüentemente capturada, como mostra o extenso material-tipo de $O$. brasiliensis que foi obtido. É possível que esta espécie se torne um problema ecológico nas regiões em que ocorre pois os representantes de Opsanus são resistentes, territoriais e agressivos (ver Gray \& Winn, 1961). Todavia, mais informações são necessárias para avaliar o status da invasão desta espécie e suas implicações nos ecossistemas marinhos brasileiros.

\section{Agradecimentos}

Nossos agradecimentos a Douglas Moreira de Souza (taxidermista do Museu de Zoologia da USP), pela preparação do esqueleto, a Eduardo Baena, pela orientação durante a tomada de fotografias dos exemplares, à Gabriela Martinhão Ignácio, pela preparação do mapa, à Fundação Boticário de Proteção à Natureza (Processo 0653_20051), pela ajuda nos projetos de ecologia realizados em Antonina, ao Conselho Nacional de Desenvolvimento Científico e Tecnológico (CNPq), e aos Drs. Flávio César Thadeo de Lima, José Lima de Figueiredo e Naércio Aquino Menezes (MZUSP), pela revisão do manuscrito.

\section{Referências Bibliográficas}

CARVALHO-FILHO, A. 1999. Peixes: costa brasileira. Editora Melro. $3^{a}$ Ed. São Paulo.

COLLETTE, B.B. 1974. A review of the coral toadfishes of the genus Sanopus with descriptions of two new species from Cozumel Island, Mexico. Proc. Biol. Soc. Wash. 87(18):185-204.

COLLETTE, B.B. 2001. Opsanus dichrostomus, a new toadfish (Teleostei: Batrachoididae) from the Western Caribbean sea and southern Gulf of Mexico. Occas. Pap. Mus. Zool. Univ. Michigan. 731:1-16.

COLLETTE, B.B. 2002. Batrachoididae. In The Living marine resources of the Western Central Atlantic. Vol 2.: Bony Fishes part 1 (Acipenseridae to Grammatidae) (K.E. Carpenter, ed.). FAO Species Identification Guide for Fishery Purposes and American Society of Ichthyologists and Herpetologists Special Publication No. 5. p. 1026-1042.

GERHARDINGER, L.C, FREITAS, M.O., ANDRADE, A.B. \& RANGEL, C.A. 2006. Omobranchus punctatus (Teleostei: Blenniidae), an exotic blenny in southwestern Atlantic. Biol. Invasions 8:941-946.

GOODE, G.B. \& BEAN, T.H. 1880. Catalogue of a collection of fishes obtained in the Gulf of Mexico. Proc. U.S. Nat. Mus. 2(98):333-345.

GRAY, G.A. \& WINN, H.E. 1961. Reproductive ecology and sound production of scarecrow toadfish, Opsanus tau. Ecology 42(2):274-282.

McEACHRAN, J.D. \& FECHHELM, J.D. 1998. Fishes of the Gulf of Mexico. Vol. 1: Myxiniformes to Gasterosteiformes. Austin. University of Texas.

MENEZES, N.A., BUCKUP, P.A., FIGUEIREDO, J.L. \& MOURA, R.L. 2003. Catálogo dos peixes marinhos do Brasil. Museu de Zoologia da Universidade de São Paulo. São Paulo.

MENEZES, N.A. \& FIGUEIREDO, J.L. 1998. Revisão das espécies da Família Batrachoididae no litoral brasileiro com a descrição de uma espécie nova (Osteichthyes, Teleostei, Batrachoidiformes). Pap. Avuls. Zool., S. Paulo 40(22):337-357.

ORSI RELINI, L. 2002. Occurrence of the South American fish Pingupes brasilianus (Pinguipedidae) in the Mediterraneum. Cybium 26(2): 156-158.

ROBINS, C.H., RAY, G.C., DOUGLASS, J. \& FREUND, R. 1986. A field guide to Atlantic coast fishes. The Petterson Field Guide Series. Houghton Mifflin Company. Boston.

ROTUNDO, M.M, SPINELLI, M. \& ZAVALA-CAMIN, L.A. 2005. Descrição de uma espécie nova de Opsanus (Teleostei - Batrachoididae) no litoral do Estado de São Paulo, Brasil. Revista Ceciliana 16(23):93-99.

SCHULTZ, L.P. \& REID, E.D. 1937. The American toadfishes of the genus Opsanus. Copeia 1937(4):211-212.

SILVA, J.S.V. \& SOUZA, R.C.C.L. 2004. Água de lastro e bioinvasão. Editora Interciência. Rio de Janeiro. 